\title{
Deployment and retrofit strategy for rubber-tyred gantry cranes considering carbon emissions
}

\author{
Yi Ding ${ }^{\mathrm{a}, \mathrm{b}}$, Yang Yang ${ }^{\mathrm{c}, *}$, Leonard Heilig ${ }^{\mathrm{b}}$, Eduardo Lalla-Ruiz ${ }^{\mathrm{d}}$, Stefan Voss ${ }^{\mathrm{b}}$ \\ ${ }^{a}$ Logistics Research Center, Shanghai Maritime University, Shanghai, China \\ ${ }^{\mathrm{b}}$ Institute of Information Systems (IWI), University of Hamburg, Hamburg, Germany \\ ${ }^{\mathrm{c}}$ Maritime Economics Research Center, University of Hamburg, Hamburg, Germany \\ d Department of Industrial Engineering and Business Information Systems, University of Twente, Enschede, the Netherlands
}

\section{A R T I C L E I N F O}

\section{Keywords:}

Green Port

Rubber-tyred Gantry Cranes

RTGs retrofitting

Yard crane deployment

Corridor Method

Integer programming

\begin{abstract}
A B S T R A C T
Driven by green port initiatives, container terminal operators have been substituting environmentally and economically inefficient diesel-powered rubber-tyred gantry cranes (RTGs) with new or retrofitted electric ones. Such an investment is closely related to operational activities, and the process of retrofitting should be carried out under the premise of satisfying the terminal yard container handling requirement. An integer programming model is proposed to help decision-makers determine the investment timing and deploy available RTGs to achieve a $\mathrm{CO}_{2}$ emissions reduction target. Considering the distinctive characteristics of different types of RTGs, we develop a deployment plan as well as an RTGs purchase and retrofit strategy. Moreover, the decisions satisfy an operation workload requirement. A tailored Corridor Method (CM) is used to accommodate large scale realworld instances. The application is illustrated with a modestly sized container terminal yard. Results confirm that electrifying diesel-powered RTGs is effective in curbing $\mathrm{CO}_{2}$ emissions and reducing energy costs. Our numerical experiments also highlight the importance of an appropriate emissions reduction target.
\end{abstract}

\section{Introduction}

Environmental impacts of ports, such as emissions from seagoing vessels and pollution caused by heavy-duty trucks and handling equipment (Geerlings, Kuipers, \& Zuidwijk, 2018), have caused serious concerns in the maritime industry around the world. Driven by the green port initiatives (e.g., China (12th and newest 13th Five-Year Plan), Europe (ESPO, 2012), Singapore (Maritime Singapore Green Initiative), and USA (Clean Ports) (Environmental Protection Agency, 2010)), container terminal operators have developed various strategies for alleviating the adverse environmental impacts. Therefore, it is essential for terminal policymakers, as a first step, to implement solutions at the terminal level to mitigate emissions. Electrifying diesel-powered rubbertyred gantry cranes (dRTGs) is considered as one of the most promising methods for energy consumption and emissions reduction in ports. Several projects have also been carried out by equipment suppliers in terminals around the world, for instance Kalmar and Vahle provide electrification technology solutions to retrofit RTGs (see, e.g., Port Technology, 2017; Port Technology, 2018). For example, 57 RTGs were changed into electric-driven RTGs (eRTGs) in 2 years in Tianjin Port, which could save the unit energy consumption of yard cranes by almost 80\% (Jinru, 2011).

The retrofitting of RTGs was first introduced in 2006 (Kuilboer, 2006), referring to the transformation of dRTGs into hybrid dieselelectric RTGs (hRTGs) or eRTGs. ERTGs are powered via electric cables instead of diesel engines (Obata et al., 2010) and some equipment suppliers also provide solutions with switchable diesel and electric power for hRTGs, such as cable reels, conductor rails using the plug-in or drive-in system, high voltage power lines and cable chains (PEMA Environment Committee, 2013). The diesel engine in an hRTG is turned off when operating, and the required power is driven by a specially designed collector to transport electricity from the electric cable. Different variants of energy storage system have been studied, such as super-capacitors (Zhao, Schofield, \& Niu, 2017), lithium batteries (Lyu, 2016), and flywheels (Flynn, McMullen, \& Solis, 2007; Flynn, Mcmullen, \& Solis, 2008).

These technologies promise to reduce operating costs by $50 \%-70 \%$, energy consumption by $10 \%-30 \%$, and noise and exhaust emissions by

\footnotetext{
* Corresponding author. Address: Moorweidenstraße 18, 20148, Hamburg, Germany

E-mail addresses: yiding@shmtu.edu.cn (Y. Ding), yang.yang@uni-hamburg.de (Y. Yang), leonard.heilig@uni-hamburg.de (L. Heilig), e.a.lalla@utwente.nl (E. Lalla-Ruiz), stefan.voss@uni-hamburg.de (S. Voss).
} 
Table 1

Energy consumption of eRTGs and dRTGs.

\begin{tabular}{ccc}
\hline & eRTGs & dRTGs \\
\hline Energy consumption (per TEU) & $2.28 \mathrm{kwh}$ & $1.27 \mathrm{~kg}$ \\
Cost of energy (USD/TEU) & 0.384 & 1.295 \\
Environmental noise pollution (Decibels) & 69.80 & 94.30 \\
\hline
\end{tabular}

* Based on an oil price of $\$ 1.020 / \mathrm{kg}$ and electricity price of $\$ 0.168 / \mathrm{kWh}$.

40\%, according to Obata et al. (2010),Vujicic, Zrnic, and Jerman (2013), and actual operation data. Table 1 summarizes the historical data in 2015 of a terminal in Shanghai Waigaoqiao Port. DRTGs consumed approximately $1.27 \mathrm{~kg}$ of diesel per twenty-foot equivalent unit (TEU), which corresponds to energy consumption of 1.851 kilograms of coal equivalent (kgce). In comparison, eRTGs consumed approximately 2.28 kWh of electricity per TEU or equivalently 0.834 kgce, $54.9 \%$ less. This leads to a reduction of $29.7 \%$ of the total energy costs. According to United Nations Conference on Trade and Development (UNCTAD), the global container throughput in 2017 hit 752 million TEU, indicating that the implementation of diesel-electric retrofitting on RTGs not only reduces terminal emissions considerably but may also save up to around $\$ 685$ million of energy costs (i.e., 752 million TEU - (\$1.295/TEU $\$ 0.384$ /TEU)).

RTGs play a significant role in yard operations. Such an implementation of the oil-electricity retrofit is highly relevant to operational activities. It should be carried out under the premise of satisfying the terminal yard container handling requirement. On account of this, a trade-off needs to be made between maintaining the appropriate or required operating capacity and investment decision implementation. However, the investment decisions of RTGs are usually made by managers based on their current practical experiences. Therefore, this study aims to contribute to the literature by providing a simple model to make integrated cost-minimizing decisions of RTGs retrofit and deployment, considering both the terminal operating efficiency and emissions. We take into account the distinctive characteristics of the three types of RTGs, i.e., purchase cost, retrofit cost, operation capability, energy consumption, and emissions. We focus on the integrated RTGs retrofit and deployment simultaneously, and the major contributions are provided as follows:

(i) An integer programming model is proposed to determine the investment timing and the deployment of available RTGs. The objective is to minimize the total costs, including both investment cost and operating cost, while reducing emissions and remaining productive.

(ii) A detailed scenario analysis of the emissions reduction target and the operation capability of RTGs is presented, which can provide a reference to terminal yard manager.

(iii) A tailored Corridor Method is designed for solving this integer programming model. Numerical experiments are conducted, based on test instances generated from real terminal operational data, to evaluate the effectiveness and efficiency of the proposed solution method.

The article is organized as follows. We give an overview of the existing literature in Section 2. Section 3 describes the RTGs management problem in detail. Section 4 presents the mathematical model of the problem. The solution approach is presented in Section 5. Section 6 reports the results of numerical experiments on a set of representative problem instances and compares the performances of two different solution methods numerically. Conclusions and an outlook for further research are given in Section 7.

\section{Literature Review}

This article is based on two different bodies of literature. The first covers the area of operational management of RTG yard cranes, which can generally be classified into two categories: (i) scheduling problem, which aims at determining the sequence of yard blocks served by each YC; (ii) deployment problem, which aims at allocating YC to different yard zones. The second body of literature is on green fleet management and to further understand how investment decisions, e.g., purchase and retrofit, affect fleet operational management.

The operational management of yard cranes (YCs) in container terminals has been well studied. Previous works on scheduling problem, e. g., Ng and Mak (2005),Liang, Gu, Lu, and Ding (2015), and Sha et al. (2017), have primarily focused on optimizing the scheduling of RTGs to minimize the sum of job waiting times or completion times. Kim and Kim (2003) considered the dispatching of a single crane to minimize the total gantry time. Several different strategies were pursued to optimize YC deployment, e.g., minimizing the total unfinished jobs (Zhang, Yw, Liu, \& Linn, 2002), minimizing the total jobs overflow (Linn \& Zhang, 2003), and minimizing the average vehicle job waiting time for YC service (Huang, Guo, \& Lau, 2011). Most of these studies on YC operational management, whether deployment or scheduling, are devoted to promoting operational efficiency.

In contrast, only a few works address the emissions reduction of terminals at the operational level, as noted in Yang and Chang (2013). He, Huang, and Yan (2015) included YC scheduling with energy consumption. Sha et al. (2017) optimized the scheduling of YC with minimal energy consumption from a low carbon perspective. Yu, Li, Sha, and Zhang (2019) considered $\mathrm{CO}_{2}$ emissions and workload delays in the deployment of RTGs simultaneously. All these works considered $\mathrm{CO}_{2}$ emissions in the operational management of RTGs but did not distinguish between different RTGs technologies.

On the other hand, green fleet management with different types of vehicles involved is referred as a supplementary source. The problem has been tackled using integer programming (Stasko \& Gao, 2010; Parthanadee, Buddhakulsomsiri, \& Charnsethikul, 2012), stochastic dynamic programming (SDP; Hsu, Li, Liu, \& Chao, 2011; Kleindorfer, Neboian, Roset, \& Spinler, 2012), approximate dynamic programming (ADP; Stasko \& Gao, 2012), stochastic programming (SP; Ansaripoor, Oliveira, \& Liret, 2014), and a multistage dynamic network (Shi, Song, \& Powell, 2014). For instance, Stasko and Gao (2012) presented an approximate dynamic programming approach for making vehicle purchase, resale, and retrofit decisions in a fleet setting with stochastic vehicle breakdowns. Kleindorfer et al. (2012) examined replacement decisions for a fleet of postal delivery vehicles with the option of adopting electric vehicles under uncertainties.

In summary, (1) few studies have addressed the operational management of YCs in a green manner; (2) there are a large number of relevant research studies on deployment problems in the literature, but to the best of our knowledge few studies have explicitly taken the distinct characteristics of different types of RTGs into account; (3) it's rare to find a truly integrated optimization of both investment and crane fleet operational management. As far as we know the closest attempt so far is Stasko and Gao (2010), which developed an integer programming method to make vehicle purchase decisions over a long time horizon, while simultaneously making vehicle task assignments at an aggregated level. Therefore, this study addresses the decisions of investment and deployment of RTGs simultaneously at a container terminal yard, while taking into consideration energy consumption and environmental emissions, which has not been attempted before. An essential aspect of this work is that we include the operation characteristics of three types of RTGs that are available on the market.

\section{Problem Description}

A container storage yard is a place to temporarily store inbound and outbound containers with four basic operations: receiving export containers from external vehicles, retrieving export and transshipment containers for loading at seaside, receiving import containers and 


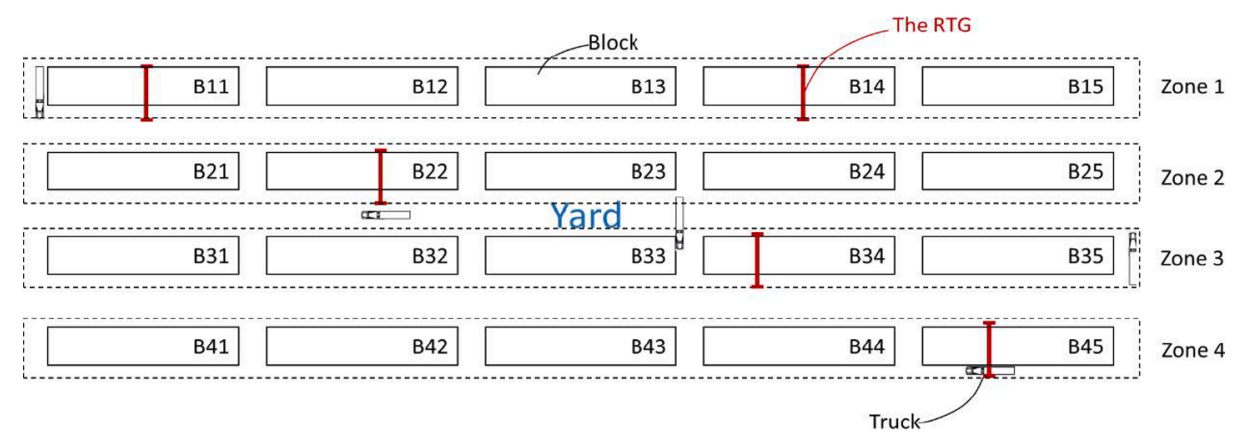

Fig. 1. Layout of container yard.

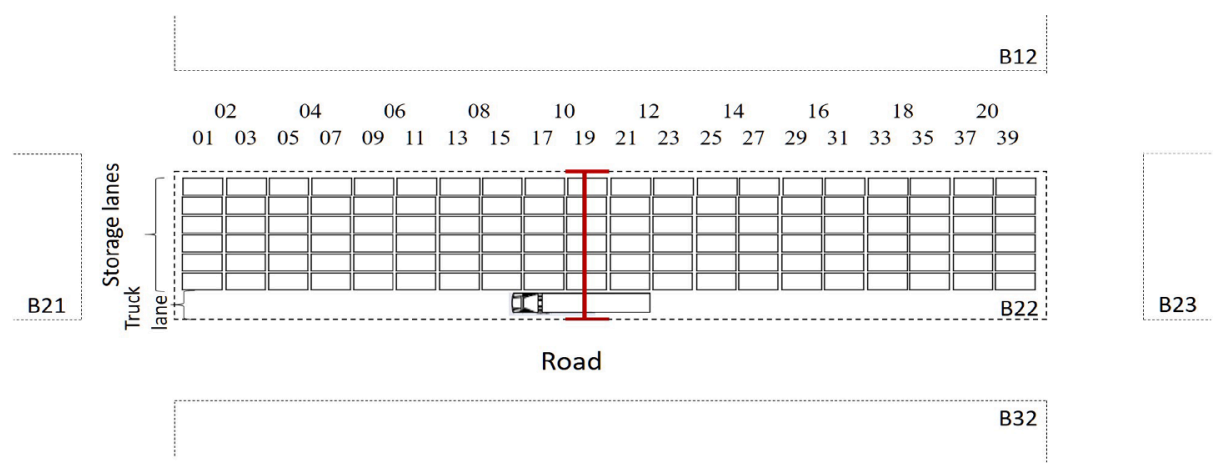

Fig. 2. Bay section of a container yard block.

transshipment containers from seaside, and retrieving import containers for pickup by external vehicles (Zhang, Zhang, Zheng, \& Miao, 2011). Fig. 1 displays the layout of a yard that is divided into blocks. One block is typically divided into seven lanes along its width - six storage lanes for stacks of containers and one lane for trucks between a pair of blocks. A block is divided along its length into bays, typically 40 in number, as depicted in Fig. 2.

RTGs need to move from blocks with surplus capacities to those in shortage because the workload distribution of a yard changes over time. As shown in Fig. 1, an intra-zone movement of an RTG from block B11 to B14 in a straight line is much easier than the movement from block B22 to B34 with two 90 degree turns, an inter-zone movement. (Zhang et al., 2002).

All types of RTGs can move along the yard lane to cover the entire zone. Once they need to transition from one block to another, the electric engines of hRTGs and eRTGs will need to be turned off, and power buffers or tailor-designed collectors will power them. After transferring to the target block, their working power will be re-switched to electricity. This transition process takes a long time. Furthermore, an eRTG usually needs to store enough energy to move from one block to another without a diesel engine (PEMA Environment Committee, 2013). Considering the reduced flexibility of eRTGs and hRTGs, it is usually assumed that both are incapable of moving from zone to zone (see in Fig. 1).

The implementation of either hRTGs or eRTGs can reduce carbon emissions and operating cost, so it seems sensible to replace all dRTGs as soon as possible. However, the main concern here is that the demands from both seaside and landside still need to be fulfilled, in the meantime these operations highly depend on the RTGs available. Thus, an appropriate or required operating capacity needs to be maintained. And with limited budgets, it is not possible to retrofit all RTGs at the same time. Moreover, it's not necessary to equip redundant RTGs if there exist any. Therefore, there is a trade-off among emissions, budgets, and yard workloads.

In practice, planners and crane operation supervisors make such decisions based on their personal experience, however it is difficult to make appropriate decisions, especially for container terminals with lots of RTGs and blocks, for instance, a yard with 100 RTGs and 70 blocks. To the best of our knowledge, monthly financial procurement plan is used in most container terminals. Thus, a model where decisions are made monthly is proposed to provide more operational level planning. We consider in total 12 periods and each period represents one month. Based on the monthly workloads in each zone, the following two sets of decisions are simultaneously considered to minimize the total costs, which consist of investment cost and operating cost:

(i) Investment: How many RTGs should be purchased or retrofitted each month under monthly budgets to reach a carbon emissions reduction target and fulfill monthly operating workloads.

(ii) Deployment: For RTGs deployment, the main concern is to ensure the number of RTGs deployed in each zone can satisfy the container handling requirement. All types of RTGs are allowed to move in an intra-zone fashion at any time. While inter-zone movement is only allowed for dRTGs, which means eRTGs and hRTGs always stay in their initial deployed zones. The movement routing of RTGs is not considered in this paper.

\section{Model development}

\subsection{Assumptions}

The RTGs management model is based on the following assumptions, which are all based on real practices in the field:

1. Only dRTGs can undergo diesel-electric retrofitting. DRTGs and eRTGs are commercially available, whereas hRTGs can only be created by retrofitting. And all types of RTGs can operate simultaneously in the same yard zone.

2. The movement cost of the intra-zone movement is ignored, because the fuel consumption cost of the inter-zone movement is much 
Table 2

Definitions of sets, parameters and decision variables.

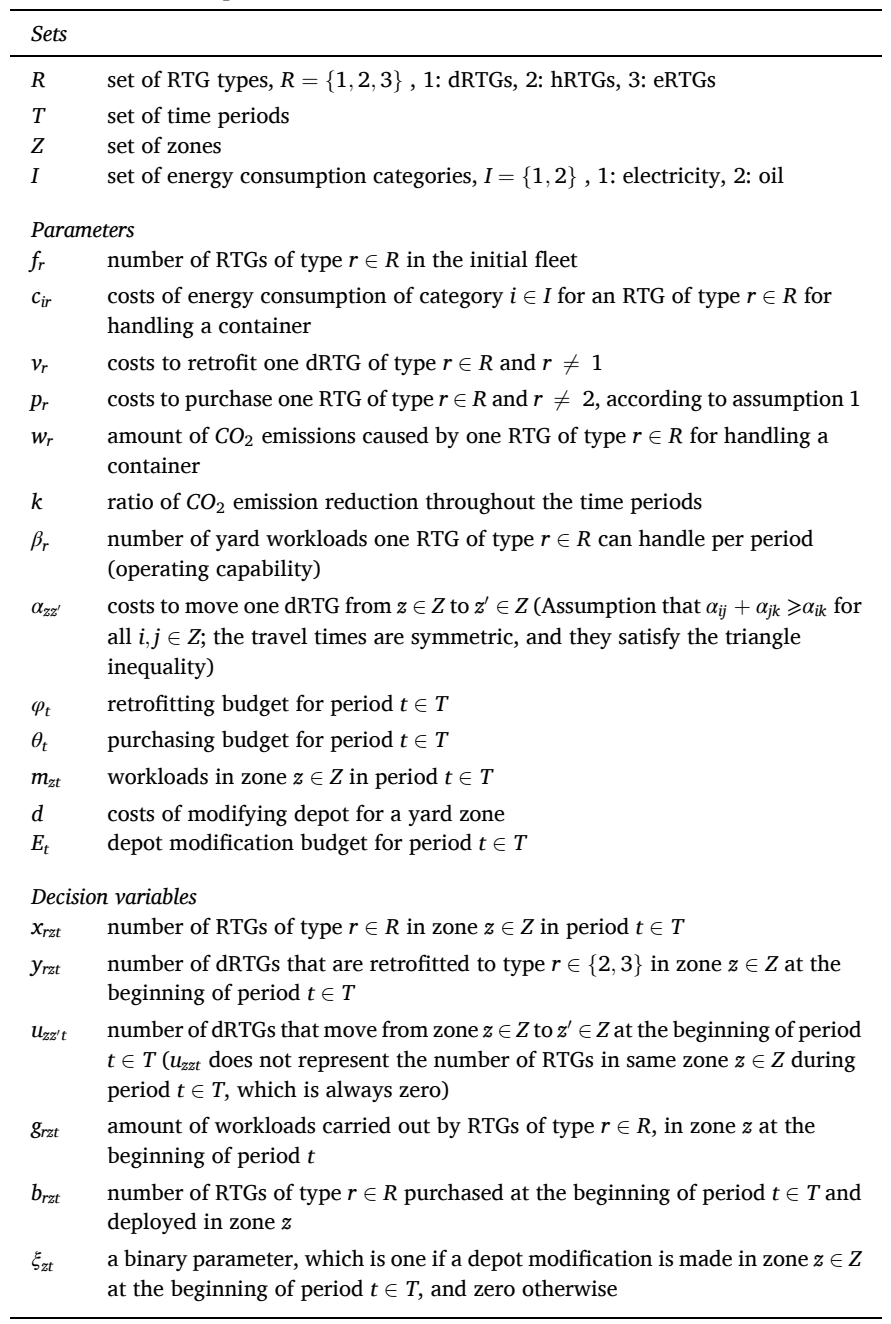

higher. Except for energy costs, all the other monthly costs of the different types of RTGs are roughly the same. Accordingly, the operating costs are defined as the diesel and electrical energy costs for handling one TEU and the inter-zone movement cost.

3. ERTGs and hRTGs are not able to move across zones but can move between intra-zone blocks.

4. The deploying, retrofitting, and purchasing of an RTG occur only at the beginning of each period. One RTG can work only in a single zone during each time period, even if it has surplus capacity.

5. Depot modification for power grid is needed in a yard zone at least one period before hRTGs and eRTGs are deployed. The modification is assumed to take one period to finish.

6. Depot modification does not affect the operations of the original equipment.

7. Compared to the operating lifetime of a terminal and service life of RTGs, monthly planning studied in this paper is rather short, so equipment retirement is ignored.

8. A monthly financial procurement plan is devised.

\subsection{Mathematical model}

In this section, we present an integer programming model. The definitions of the sets, parameters, and decision variables are given in Table 2.

The mathematical formulation consisting of RTGs purchasing, retrofitting, and deployment decisions is stated as follows.

$$
\begin{aligned}
& \min \sum_{t \in T}\left(d \sum_{z \in Z} \xi_{z t}+\sum_{z \in Z} \sum_{z^{\prime} \in Z_{z} z \neq z^{\prime}}\left(u_{z z^{\prime} t} \alpha_{z z^{\prime}}\right)+\sum_{r \in R}\left(\sum_{i \in I}\left(c_{i} r \sum_{z \in Z} g_{r z t}\right)+p_{r} \sum_{z \in Z} b_{r z t}\right.\right. \\
& \left.\left.\quad+v_{r} \sum_{z \in Z} y_{r z t}\right)\right)
\end{aligned}
$$

Subject to:

$$
\begin{aligned}
& \sum_{z \in Z} x_{r z 1}=f_{r}+\sum_{z \in Z} b_{r z 1}-\sum_{z \in Z} y_{r z 1}, \forall r \in R \\
& x_{r z t}=x_{r z(t-1)}+b_{r z t}+y_{r z t}, \quad y_{r z t}= \begin{cases}y_{r z t} & \text { if } \sum_{t^{\prime}=1}^{t-1} \xi_{z t^{\prime}}=1 \\
0, & \text { otherwise }\end{cases} \\
& b_{r z t}= \begin{cases}b_{r z t} & \text { if } \sum_{t^{\prime}=1}^{t-1} \xi_{z t^{\prime}}=1 \\
0, & \text { otherwise }\end{cases} \\
& \forall r \in\{2,3\}, \forall z \in Z \\
& \forall t \in T: t \neq 1
\end{aligned}
$$

$$
\begin{array}{r}
x_{1 z t}=x_{1 z(t-1)}+b_{l z t}-\sum_{r \in\{2,3\}} y_{r z t}+\sum_{z^{\prime} \in Z: z^{\prime} \neq z}\left(u_{z^{\prime} z t}-u_{z z^{\prime} t}\right), \\
y_{r z t}=\left\{\begin{array}{l}
y_{r z t} \text { if } \sum_{t^{\prime}=1}^{t-1} \xi_{z t^{\prime}}=1 \\
0, \text { otherwise }
\end{array}\right. \\
\forall z \in Z, \forall t \in T: t \neq 1
\end{array}
$$

$$
\begin{aligned}
& \beta_{r} x_{r z t} \geqslant g_{r z t}, \quad \forall r \in R, \forall z \in Z, \forall t \in T \\
& \sum_{r \in R} g_{r z t}=m_{z t}, \quad \forall z \in Z, \forall t \in T \\
& \sum_{r \in\{2,3\}} \sum_{z \in Z} p_{r} b_{r z t} \leqslant \theta_{t}, \quad \forall t \in T \\
& \sum_{z \in Z} \sum_{r \in\{2,3\}} v_{r} y_{r z t} \leqslant \varphi_{t}, \quad \forall t \in T
\end{aligned}
$$

$\sum_{z \in Z} d \xi_{z t} \leqslant E_{t}, \quad \forall t \in T$

$\sum_{t \in T} \xi_{z t} \leqslant 1, \quad \forall z \in Z$

$\sum_{z \in Z} u_{z z t}=0, \quad \forall t \in T$

$\sum_{t \in T} \sum_{r \in R}\left(w_{r} \sum_{z \in Z} g_{r z t}\right) \leqslant w_{1}(1-k) \sum_{t \in T} \sum_{z \in Z} m_{z t}$

$x_{r z t}, y_{r z t}, u_{z z^{\prime} t}, g_{r z t}, b_{r z t} \in \mathbb{N}$,

$\xi_{z t} \in\{0,1\}$,

$\forall z, z^{\prime} \in Z, \forall t \in T, \forall r \in R$

The objective function is to minimize the total costs, including investment costs (retrofit, purchase, and depot modification costs) and operating costs (energy consumption of crane inter-zone movement and average operation energy costs per TEU). Constraints (2)-(4) consider the conservation of flow for RTGs. Constraints (2) specify the number of dRTGs in each zone from the first period according to the assumptions. Constraints (5) and (6) ensure that the deployed RTGs can handle the workloads in all the zones in each period. Constraints (7)-(9) impose the purchasing, retrofitting, and depot modification capital budget constraints in each period. Constraint (10) ensures that depot modification can be done only once for each yard zone. Constraint (11) prohibits dRTGs from moving within the same zone. Constraint (12) forces the $\mathrm{CO}_{2}$ emissions to decrease by a factor of $k$ throughout the entire 
planning horizon. Constraints (13) defines the nature of the variables.

Theoretically, the yard crane deployment IP models can be solved by exact methods like the cutting-plane method, using standard software such as Cplex (Zhang et al., 2002). Models with a small number of variables can be solved within seconds or minutes (Linn \& Zhang, 2003). When model size increases, however, computation time grows to hours or even days. Consequently, a fast heuristic algorithm is necessary to solve this problem.

\section{Optimization Framework}

The optimization framework consists of the CM algorithm and the modified mathematical model used by the algorithm to retrieve solutions to the subproblems of the original model defined in the last section. Corridors around solutions are first defined in Section 5.2. They are used to modify the original model by adding corridors as additional constraints and adapting the original objective function.

\subsection{Corridor Method}

Small instances of the mathematical model formulated in Section 4 can easily be solved by an IP solver. When the size increases, it fails to find optimal solutions within a reasonable time. A common way to solve such a problem is to relax some constraints and decompose the problem into several subproblems, which can be solved more easily. A tailored Corridor Method (CM) has been applied in different settings, for example, production planning problems (Caserta, Ramirez, \& Voß, 2010; Caserta, Voß, \& Sniedovich, 2011) and block relocation problems (Caserta et al., 2011).

The core concept of CM is the iterative definition of subproblems of the original problem by imposing extra constraints (Sniedovich \& Voß, 2006). Consequently, the optimal solution can be found in a limited part of the available space by an exact method $M$. If an improved solution is found, then a new corridor is defined around it, and the step is repeated. Otherwise, the corridor will be adjusted, either by expanding or generating a new one from a new incumbent solution in the pool of solutions. When all the solutions in the solution pool have been used to establish a new corridor, or otherwise specified stopping criterion applies, such as a maximum running time, the algorithm is terminated (Caserta \& Voß, 2013). Although CM is a heuristic method and cannot guarantee optimal solutions in general, it can easily be applied to the proposed model with little modification and improve the solving efficiency.

$$
\begin{aligned}
& \min \sum_{t \in T}\left(d \sum_{z \in Z} \xi_{z t}+\sum_{z \in Z} \sum_{z^{\prime} \in Z: z \neq z^{\prime}}\left(u_{z z^{\prime} t} \alpha_{z z^{\prime}}\right)+0 \cdot \sum_{z \in Z} \sum_{r \in R} j_{r z t}+0 \cdot \sum_{z \in Z} \sum_{r \in R} h_{r z t}\right. \\
& \left.+0 \cdot \sum_{z \in Z} \sum_{z^{\prime} \in Z} l_{z z^{\prime} t}+0 \cdot \sum_{z \in Z} \sum_{z^{\prime} \in R} n_{z z^{\prime} t}+\sum_{r \in R}\left(\sum_{i \in I}\left(c_{i r} \sum_{z \in Z} g_{r z t}\right)+p_{r} \sum z \in Z b_{r z t}+v_{r} \sum z \in Z y_{r z t}\right)\right)
\end{aligned}
$$

In order to limit the solution space, the deployment variables $x_{r z t}$ and $u_{z z^{\prime} t}$ are taken as corridor variables and two corridor parameters are defined, namely $\delta_{1}, \delta_{2} \geqslant 0$. Parameter $\delta_{1}$ defines the corridor around $x_{r z t}$, such that $x_{r z t}^{s} \pm \delta_{1}$. Parameter $\delta_{2}$ defines the corridor around $u_{z z^{\prime} t}$, such that $u_{z z^{\prime} t}^{s} \pm \delta_{2}$. and $d\left(u, u^{s}\right)$ defined by $x^{s}, u^{s}, \delta_{1}$ and $\delta_{2}$. We define $d\left(x, x^{s}\right), d\left(u, u^{s}\right)$, and $N_{\delta}\left(x^{s}\right.$, $\left.u^{s}\right)$ in Eqs. (14)-(16).

$$
\begin{aligned}
& d\left(x, x^{s}\right)=\sum_{r=1}^{R} \sum_{z=1}^{Z} \sum_{t=1}^{T}\left|x_{r z t}-x_{r z t}^{s}\right| \leqslant \delta_{1} \\
& d\left(u, u^{s}\right)=\sum_{z=1}^{Z} \sum_{z^{\prime}=1}^{Z} \sum_{t=1}^{T}\left|u_{z z^{\prime} t}-u_{z z^{\prime} t}^{s}\right| \leqslant \delta_{2} \\
& N_{\delta}\left(x^{s}, u^{s}\right)=\left\{(x, y, b, u, g, \xi) \in X: d\left(x, x^{s}\right) \leqslant \delta_{1}, d\left(u, u^{s}\right) \leqslant \delta_{2}\right\}
\end{aligned}
$$

Eq. (16) limits the solution space based on the incumbent solution $\mathrm{sol}^{\mathrm{s}}$ under given parameter values $\delta_{1} \in[0, \Pi \cdot|R| \cdot|Z| \cdot|T|]$ and $\delta_{2} \in[0, \Pi \cdot|Z| \cdot|Z| \cdot|T|]$, where the value of $\Pi$ is the possible maximum number of RTGs for each yard zone. When both $\delta_{1}$ and $\delta_{2}$ equal to zero, the corridor is made up of all the solutions that can be obtained with a fixed RTG number established by $x^{s}$ and $u^{s}$. Conversely, when $\delta_{1}=\max \left\{\delta_{1}\right\}$ and $\delta_{2}=\max \left\{\delta_{2}\right\}$, the corridor is the same as the original solution space $X$. Consequently, the size of $N_{\delta}\left(x^{s}, u^{s}\right)$ can be made arbitrarily small by changing the values of $\delta_{1}$ and $\delta_{2}$.

Finally, Eqs. (14) and (15) are rewritten as linear expressions with auxiliary decision variables $j_{r z t}, h_{r z t}, l_{z z^{\prime} t}$, and $n_{z z^{\prime} t}$ defined in Eqs. (17)(21).

$$
\begin{aligned}
& \sum_{r=1}^{R} \sum_{z=1}^{Z} \sum_{t=1}^{T}\left(x_{r z t}-x_{r z z}^{s}+j_{r z t}\right)=\delta_{1} \\
& \sum_{r=1}^{R} \sum_{z=1}^{Z} \sum_{t=1}^{T}\left(x_{r z t}-x_{r z t}^{s}+h_{r z t}\right)=\delta_{1} \\
& \sum_{z=1}^{Z} \sum_{z^{\prime}=1}^{Z} \sum_{t=1}^{T}\left(u_{z z^{\prime} t}-u_{z z^{\prime} t}^{s}+l_{z z^{\prime} t}\right)=\delta_{2} \\
& \sum_{z=1}^{Z} \sum_{z^{\prime}=1}^{Z} \sum_{t=1}^{T}\left(u_{z z^{\prime} t}-u_{z z^{\prime} t}^{s}+n_{z z^{\prime} t}\right)=\delta_{2} \\
& j_{r z t}, h_{r z t}, l_{z z^{\prime} t}, n_{z z^{\prime} t} \in \mathbb{R}
\end{aligned}
$$

Moreover, the objective function from Eq. (1) is adjusted as follows. A neighborhood solution $N_{\delta}\left(x^{s}, u^{s}\right)$ around $s o l^{s}$ is limited by distances $d\left(x, x^{s}\right)$

\subsection{Definition of corridors}

As explained in Section 5.1, a corridor limits the solution space by defining a smaller subproblem that can be solved more easily. $X$ denotes the feasible space of the original problem, sol $=(x, y, b, u, g, \xi) \in X$, defined by the constraints (2) to (13), and $M$ is the exact method used by an IP solver. The pool of incumbent solutions $\Omega$ can be obtained rapidly by $M$ with Cplex Branch and Cut IP solver under appropriate gap. An incumbent solution $\left(x^{s}, u^{s}\right)$ from $\Omega$ is defined as $s o l^{s}=(x, y, b, u, g, \xi)^{s}$, where the decision variables' values are $x_{r z t}^{s}\left(x^{s}\right), y_{r z t}^{s}\left(y^{s}\right), b_{r z t}^{s}\left(b^{s}\right), u_{z z^{\prime} t}^{s}$ $\left(u^{s}\right), g_{r z t}^{s}\left(g^{s}\right)$, and $\xi_{z t}^{s}\left(\xi^{s}\right)$.
By finding a maximum distance rather than explicitly fixing a subset of variables to given values (i.e., $\delta_{1}$ and $\delta_{2}$ ), a certain part of the incumbent solution is maintained. Therefore, the number of available RTGs is limited and the IP solver can choose the number of RTGs to be retrofitted, deployed, and purchased.

\subsection{CM algorithm}

After defining the corridors and adapting the mathematical formulation, we explain the steps for applying the proposed CM algorithm as 
follows. The pseudocode of the proposed CM algorithm is presented in Algorithm 1.

Algorithm 1. tailored CM algorithm

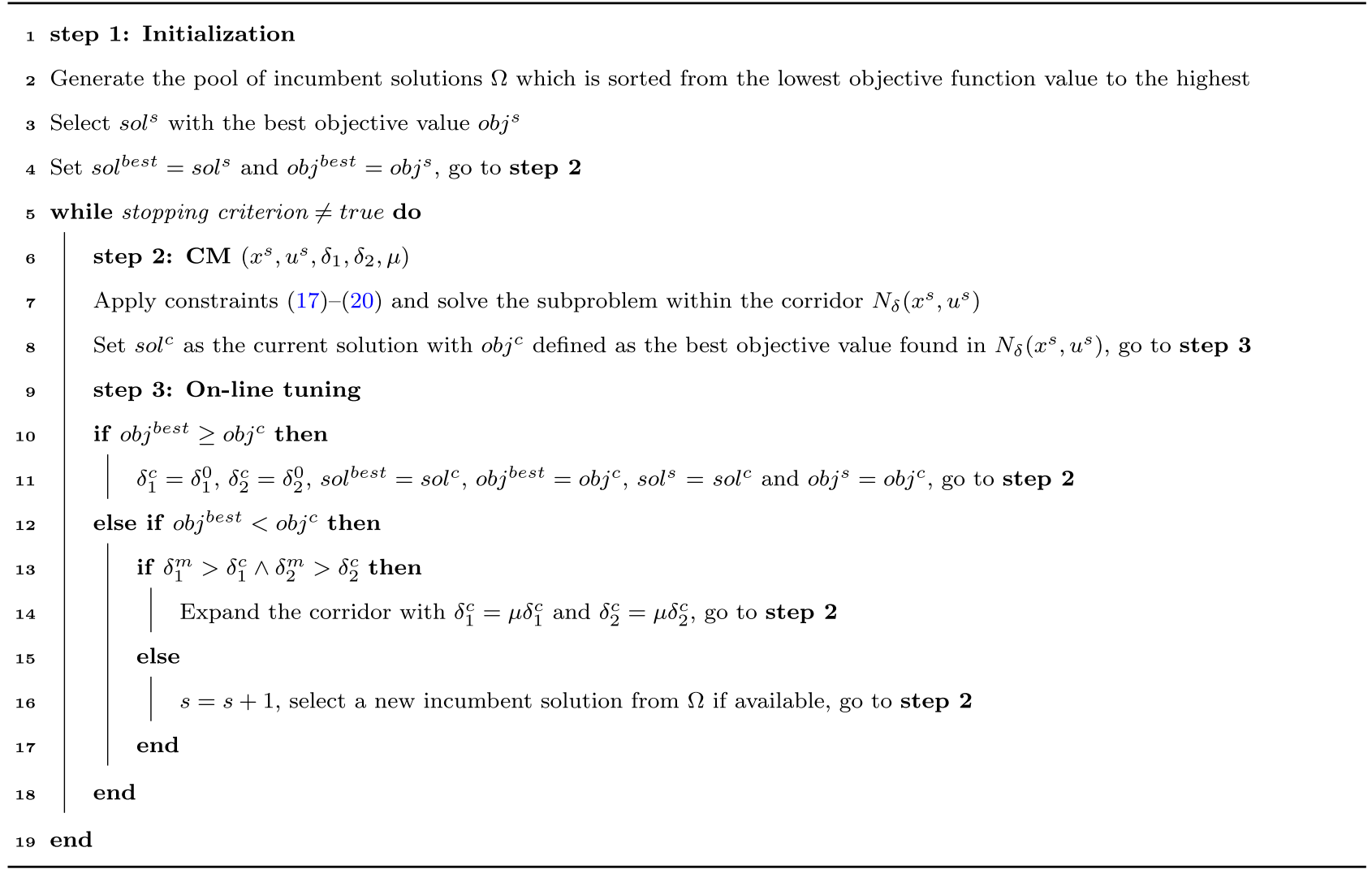

The algorithm starts with an initialization phase (step 1). The pool of incumbent solutions $\Omega$ is obtained from $M$ and sorted by value of the objective function in ascending order, whereas, as mentioned before, $M$ is the exact method used by an IP solver. The selected incumbent solution from $\Omega$ is defined as $s o l^{s}$ with objective value $o b j^{s}$. This solution is used to initiate the best solution $s o l^{\text {best }}=(x, y, b, u, g, \xi)^{\text {best }}$ and its objective value $o b j^{\text {best }}$.

Constraints specified by Eqs. (17)-(21) are added into the original IP model to define subproblems (step 2). The adapted objective function (Eq. 22) is solved, possibly to optimality, using an IP solver. If the objective value of the retrieved solution $\left(o b j^{c}\right)$ is better than the objective value of the best-known solution $\left(o b j^{\text {best }}\right.$ ), the corridor is updated to its initial value and the current solution is set as the new best-known solution. Otherwise, the corridor is expanded, and a new incumbent solution is chosen from $\Omega$, before continuing with step 2 .

The corridors are updated as follows. According to the results of each iteration, new values for $\delta_{1}$ and $\delta_{2}$ are determined in the on-line tuning phase (step 3). In each iteration, the values of $\delta_{1}$ and $\delta_{2}$ are updated in the range $\left[\delta_{1}^{0}, \delta_{1}^{m}\right]$ and $\left[\delta_{2}^{0}, \delta_{2}^{m}\right]$, respectively, whereas $\delta_{1}^{0}$ and $\delta_{2}^{0}$ are the initial values and $\delta_{1}^{m}, \delta_{2}^{m}$ are the maximum values of the parameter. The following two cases are considered.

(i) $o b j^{\text {best }}<o b j^{c}$ : No solution of lower costs has been found within $N_{\delta}\left(x^{s}, u^{s}\right)$. The corridor must be expanded to increase the probability of finding a better solution. Two cases are possible.

(a) If $\delta_{1}^{m}>\delta_{1}^{c} \wedge \delta_{2}^{m}>\delta_{2}^{c}$ then increase the value of $\delta_{1}$ between $\left[\delta_{1}^{0}\right.$, $\left.\delta_{1}^{m}\right]$, as well as $\delta_{2}$ between $\left[\delta_{2}^{0}, \delta_{2}^{m}\right]$, for example, $\delta_{1}^{c}=\mu \delta_{1}^{c}, \delta_{2}^{c}=$ $\mu \delta_{2}^{c}$, where the parameter $\mu$ defines a fixed step-up size for expanding the corridor. And repeat the $\mathrm{CM}$ phase with the expanded corridor.

(b) Else: The value of $\delta$ is already at its upper limit. Then a new incumbent solution is selected from $\Omega$ if available without adjusting the corridor.

(ii) $o b j^{\text {best }} \geqslant o b j^{c}$ : A superior solution has been found within $N_{\delta}\left(x^{s}, u^{s}\right)$. Similar to a hill-climbing search approach, $\delta_{1}^{c}=\delta_{1}^{0}, \delta_{2}^{c}=\delta_{2}^{0}$, $\operatorname{sol}^{b^{b e s t}}=\operatorname{sol}^{c}\left(s^{c} l^{c}=(x, y, b, u, g, \xi)^{c}\right), o b j^{b e s t}=o b j^{c}, s o l^{s}=s o l^{c}$, and $o b j^{s}=o b j^{c}$ are set, before a new solution is generated by solving another subproblem in step 2.

The search process (steps 2 and 3) are repeated until a pre-specified stopping criterion, e.g., the maximum number of iterations or the maximum running time, is met (line 5).

\section{Computational experiments}

In this section, we present the results of the computational experiments conducted using the proposed CM framework, which includes an analysis of cost-minimizing strategies for purchasing, deploying, and retrofitting RTGs and the impact of emissions targets and operating capabilities on decision-making. At the end of this section, the results from the algorithmic framework are compared with those from a general purpose IP solver to analyze their performances in terms of solution quality and computational time.

For solving the mathematical model proposed in Section 4, we use Cplex 12.1. The proposed CM framework (see in Section 5), 
implemented in C\#, is integrated with the Cplex implementation and uses the same computational environment. The computational experiments were conducted on a $2.60 \mathrm{GHz}$ Intel Core laptop, with a total memory of $8 \mathrm{~GB}$.

\subsection{Related Data}

Based on expert advice, the operating capabilities of hRTGs and eRTGs are set to be $80 \%$ and $65 \%$ respectively, of the capabilities of dRTGs. Only dRTGs can be retrofitted, and retrofitting costs are $\$ 320,000$ and $\$ 240,000$ for eRTGs and dRTGs, respectively, as shown in Table 3. Both eRTGs and hRTGs are assumed not to be able to move across zones. Table 4 presents the retrofit and depot modification budgets based on a monthly financial procurement plan.

A modestly sized container terminal yard with five zones, each with five blocks, is used as an example. The initial 20 RTGs are all dRTGs; the other two types of RTGs cannot be used before necessary depot modifications. Both dRTGs and eRTGs are available for purchase. Workloads are assumed based on real yard operational data, as shown in Table 5 . Moreover, the estimated costs of crane moves between yard zones have been given in Table 6, based on data from the terminal yard, which includes the actual distances between zones.

The $\mathrm{CO}_{2}$ emission factors of three types of RTGs per TEU $\left(w_{r}\right)$ are shown in Table 8, calculated based on the following emission factors for diesel and electricity, according to China Energy Statistics Yearbook 2014 (China's National Bureau of Statistics, 2015):

(i) Emission factors for diesel: the low heat value of diesel that is used in China is $42,652 \mathrm{~kJ} / \mathrm{kg}$. The default $\mathrm{CO}_{2}$ emissions factor for 1 $\mathrm{kg}$ of greenhouse gas per TJ is 74,100, taken from the 2006 IPCC Guidelines for National Greenhouse Gas Inventories. Based on the assumption that the oxidation rate of diesel is $100 \%$, a diesel density of $0.85 \mathrm{~kg} / \mathrm{l}$ yields a $\mathrm{CO}_{2}$ emission factor as $2.65 \mathrm{~kg} / \mathrm{l}$.

(ii) Emission factors for electricity: Table 7 presents base emission factors of power grids in various regions. The terminal is located in the eastern China region. The emission factor for electricity herein is $0.8095 \mathrm{kgCO}_{2} / \mathrm{kWh}$.

\subsection{Experimental results}

\subsubsection{Cost-minimizing strategies for the RTGs fleet}

According to the Chinese State Council (2016), the key objectives by 2020 are to lower carbon dioxide emission per unit of GDP by $18 \%$ from the 2015 emission level. Reducing terminal emissions is a daunting task, given the inevitable growth in throughput. A stricter $\mathrm{CO}_{2}$ emission reduction target (i.e., $k$ ) is set to be 0.25 . The fixed step-up parameter $\mu$ is set to 1.25. Cost-effective strategies are shown in Fig. 3. The comparison between no $\mathrm{CO}_{2}$ emissions limitation $(k=0)$ and $k=0.25$ is presented in Table 9. It can be observed that four zones (80\%) undergo depot modification, three new eRTGs are purchased, and $18 \mathrm{dRTGs} \mathrm{(72 \% )} \mathrm{are}$ retrofitted to meet a $25 \%$ emission reduction target. The purchase and retrofitting RTGs significantly increase the total cost (TC); however, energy cost (EC) is greatly reduced by $40 \%$, and the investment will be returned in around 15 years $^{1}$.

$T_{\text {payback }}=\frac{T C_{k=0.25}-T C_{k=0}}{E C_{k=0}-E C_{k=0.25}}=14.95($ years $)$

\subsubsection{Effect of $\mathrm{CO}_{2}$ emissions reduction target on strategies}

A sensitivity analysis is performed to evaluate the effect of $\mathrm{CO}_{2}$ emissions reduction target on investment and deployment decisionmaking. Fig. 4 plots the results of comparisons. It shows that the value of TC increases, and the EC decreases with the growth of the $\mathrm{CO}_{2}$

\footnotetext{
${ }^{1}$ The time value of money is not taken into account in this paper.
}

Table 3

Cost and capacity of RTGs.

\begin{tabular}{cccc}
\hline & dRTGs & eRTGs & hRTGs \\
\hline Diesel costs (USD/TEU) & 1.295 & 0 & 0.650 \\
Electricity costs (USD/TEU) & 0 & 0.384 & 0.214 \\
Purchase price (USD) & 800,000 & 960,000 & $/$ \\
Retrofit costs (USD) & $/$ & 240,000 & 160,000 \\
Depot modification (USD/Zone) & 0 & 960,000 & 960,000 \\
Capacity (TEU/month) & 6,000 & 3,900 & 4,800 \\
\hline
\end{tabular}

Table 4

Retrofit and depot modification budget.

\begin{tabular}{rccc}
\hline & $\begin{array}{c}\text { Retrofit } \\
\text { budget }\end{array}$ & $\begin{array}{c}\text { Depot modification } \\
\text { budget }\end{array}$ & $\begin{array}{l}\text { Purchase } \\
\text { budget }\end{array}$ \\
\hline $\begin{array}{r}\text { Monthly } \\
\text { (USD) }\end{array}$ & $1,600,000$ & $4,800,000$ & $3,200,000$ \\
\hline
\end{tabular}

Table 5

Workloads in five zones in October 2017 (in TEUs).

\begin{tabular}{cccccc}
\hline Time $(t)$ & Zone1 & Zone2 & Zone3 & Zone4 & Zone5 \\
\hline 1 & 23414 & 22702 & 21662 & 22530 & 23173 \\
2 & 23348 & 19767 & 23030 & 23886 & 22494 \\
3 & 23349 & 21913 & 23853 & 21614 & 21806 \\
4 & 22270 & 21573 & 23404 & 21043 & 23646 \\
5 & 23326 & 21985 & 22299 & 23420 & 22646 \\
6 & 22485 & 22786 & 21369 & 23400 & 23046 \\
7 & 22103 & 22674 & 24460 & 23095 & 22207 \\
8 & 21796 & 24944 & 25050 & 23496 & 21449 \\
9 & 24037 & 22428 & 23120 & 24082 & 23171 \\
10 & 22154 & 22749 & 22010 & 23970 & 22937 \\
11 & 22827 & 21430 & 24197 & 22431 & 24199 \\
12 & 21791 & 22522 & 22407 & 23809 & 23801 \\
\hline
\end{tabular}

Table 6

Cost of crane Move between zones.

\begin{tabular}{cccccc}
\hline Transition cost & & & & & \\
\hline (160 USD/move) & 1 & 2 & 3 & 4 & 5 \\
\hline 1 & 0 & 1 & 2 & 2 & 2.5 \\
2 & 1 & 0 & 2 & 2 & 2.5 \\
3 & 2 & 2 & 0 & 1 & 2 \\
4 & 2 & 2 & 1 & 0 & 2 \\
5 & 2.5 & 2.5 & 2 & 2 & 0 \\
\hline
\end{tabular}

emissions limits; however when $k$ ranges from 0.25 to 0.30 , the payback period increases, indicating that a strict limit for $\mathrm{CO}_{2}$ emissions but not always appears financially appealing. These results can provide vital information for terminal operators, i.e., it's necessary to select an appropriate $\mathrm{CO}_{2}$ emissions reduction target $(k)$ due to limited resources. It is worth mentioning that when $k$ is set higher than $40 \%$, the model is unsolvable. The reason is that the limited operating capabilities of hRTGs and eRTGs cannot satisfy the terminal workload.

\subsubsection{Effect of eRTG operation capability on strategies}

In order to evaluate the effect on decision-making, the proposed model was run with a range of eRTG operating capabilities. Table 10 displays the result, which is not surprising in that, with the increase of eRTG operation capacity from 3900 to $8000 \mathrm{TEU} / \mathrm{month}$, the payback period shrinks around $50 \%$, indicating that great importance should be attached to the improvement of operation capability. However, such an improvement leads to the rise of EC and fewer eRTGs to be deployed, when the operating capability of an eRTG is higher than or equal to that of a dRTG. The driving force behind this is a reasonable trade-off between emissions reduction and investment cost. 
Table 7

Base emission factors for power grids in different regions.

\begin{tabular}{|c|c|c|c|c|c|c|}
\hline Region of China & North & Northeast & East & Central & Northwest & South \\
\hline $\begin{array}{c}\text { Emission factor } \\
\left(\mathrm{kgCO}_{2} / k W h\right)\end{array}$ & 1.0580 & 1.1281 & 0.8095 & 0.9724 & 0.9578 & 0.9183 \\
\hline
\end{tabular}

Table 8

$\mathrm{CO}_{2}$ emission factors of RTGs per TEU.

\begin{tabular}{lccc}
\hline & dRTGs & eRTGs & hRTGs \\
\hline $\mathrm{CO}_{2}$ emission factor $\left(\mathrm{kgCO}_{2} / \mathrm{TEU}\right)$ & 3.363 & 1.8468 & 2.4793 \\
\hline
\end{tabular}

\subsection{Performance of the Corridor Method}

The effectiveness of the proposed CM algorithm is evaluated for yard sizes from two to ten zones. Table 11 presents the summary statistics for each instance implemented in both Cplex and the CM approach. Column 1 to Column 4 show the problem size. $N_{Z}$ represents the number of zones. Column 2, 3, and 4 show the number of binary and inter variables $\left(N_{B}\right.$ and $\left.N_{I}\right)$ and the "Rows-Columns-Nonzeros" of each instance is obtained from CPLEX after presolve is performed. The column Cplex contains the solution $O b j$. provided by the IP solver and the relative error provided by Cplex (Gap (\%)). The column CM includes the solution $O b j$. provided by the proposed $\mathrm{CM}$ algorithm as well as the relative error with respect to the Cplex solution $\left(R E=\frac{C M(O b j .)-C p l e x(O b j .)}{C p l e x(O b j .)}\right)$.

The following conclusions are drawn:

1. The computational time of Cplex increased with the number of zones. It fails to solve to optimality within a reasonable time when the number of zones reaches 10 .

2. The $\mathrm{CM}$ algorithm yielded non-inferior solutions within a reasonable time, though it takes more time for small instances. The mean CPU time was $578 \mathrm{~s}$. The deviation of the CM solutions $R E$ ranged from $-0.66 \%$ to $0.06 \%$, which means CM is competitive, especially for a large terminal yard.

\section{Conclusion}

Driven by the green port initiatives, this paper focuses on the implementation decisions of electrifying dRTGs in the container terminal yard to mitigate emissions. Past studies have investigated RTGs deployment problems and operational management in the field of fleet operations. However, there is few literature available on crane fleet

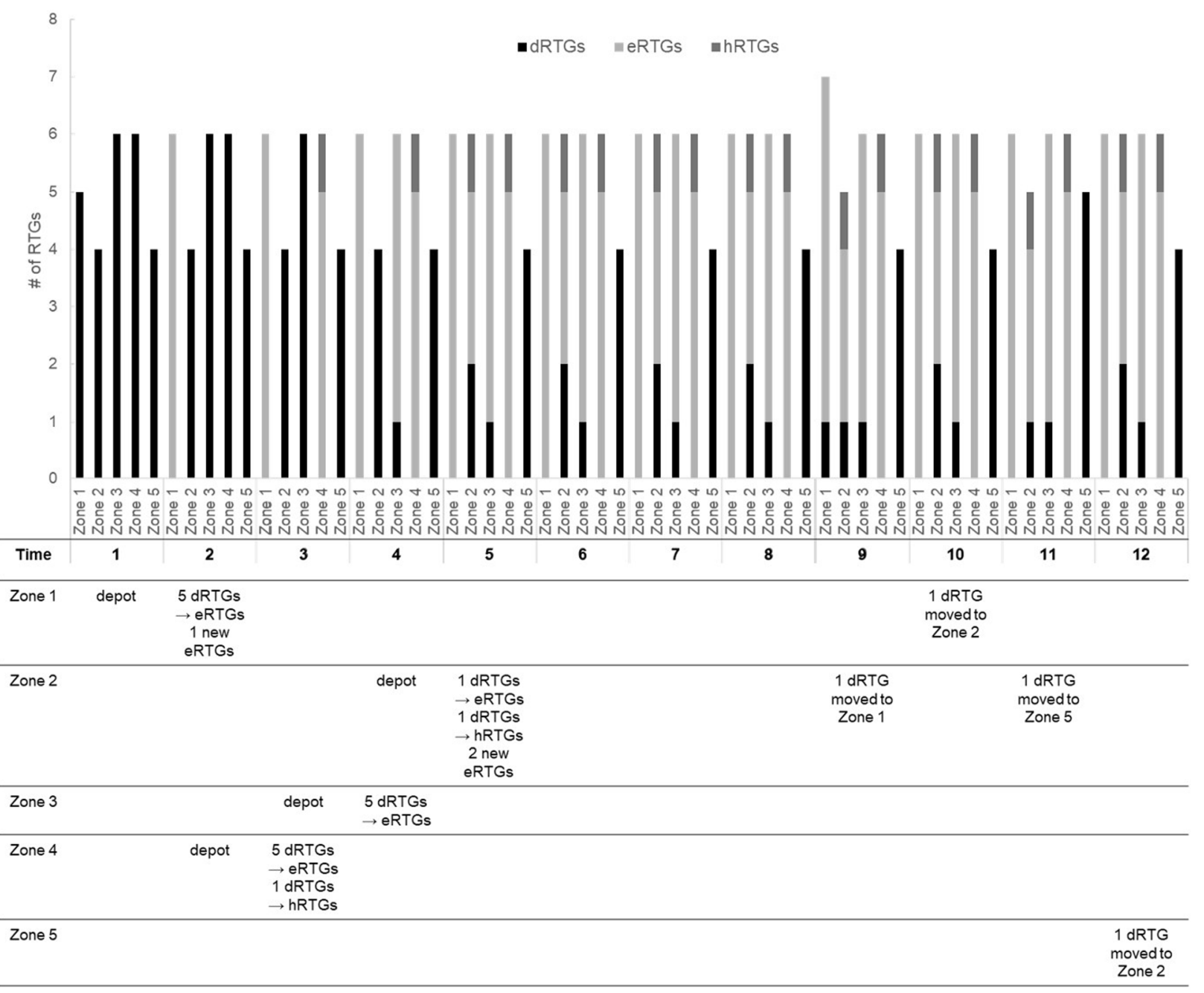

Fig. 3. Cost-effective strategies when $k=0.25$. 
Table 9

Comparison between $k=0$ and $k=0.25$.

\begin{tabular}{|c|c|c|c|c|c|c|c|}
\hline$k$ & $\begin{array}{l}\text { Total costs } \\
\text { (TC) }\end{array}$ & $\begin{array}{l}\text { Energy costs } \\
\text { (EC) }\end{array}$ & $\begin{array}{c}\text { Number } \\
\text { of } \\
\text { dRTGs }\end{array}$ & $\begin{array}{l}\text { Number } \\
\text { of } \\
\text { hRTGs }\end{array}$ & $\begin{array}{c}\text { Number } \\
\text { of } \\
\text { eRTGs }\end{array}$ & $\begin{array}{c}\text { Total } \\
\text { number } \\
\text { of RTGs }\end{array}$ & $\begin{array}{c}\text { Number of } \\
\text { depot } \\
\text { modifications }\end{array}$ \\
\hline 0 & $1,641,073$ & $1,641,073$ & 25 & 0 & 0 & 25 & 0 \\
\hline 0.25 & $11,181,088$ & $1,001,452$ & 7 & 2 & 19 & 29 & 4 \\
\hline
\end{tabular}

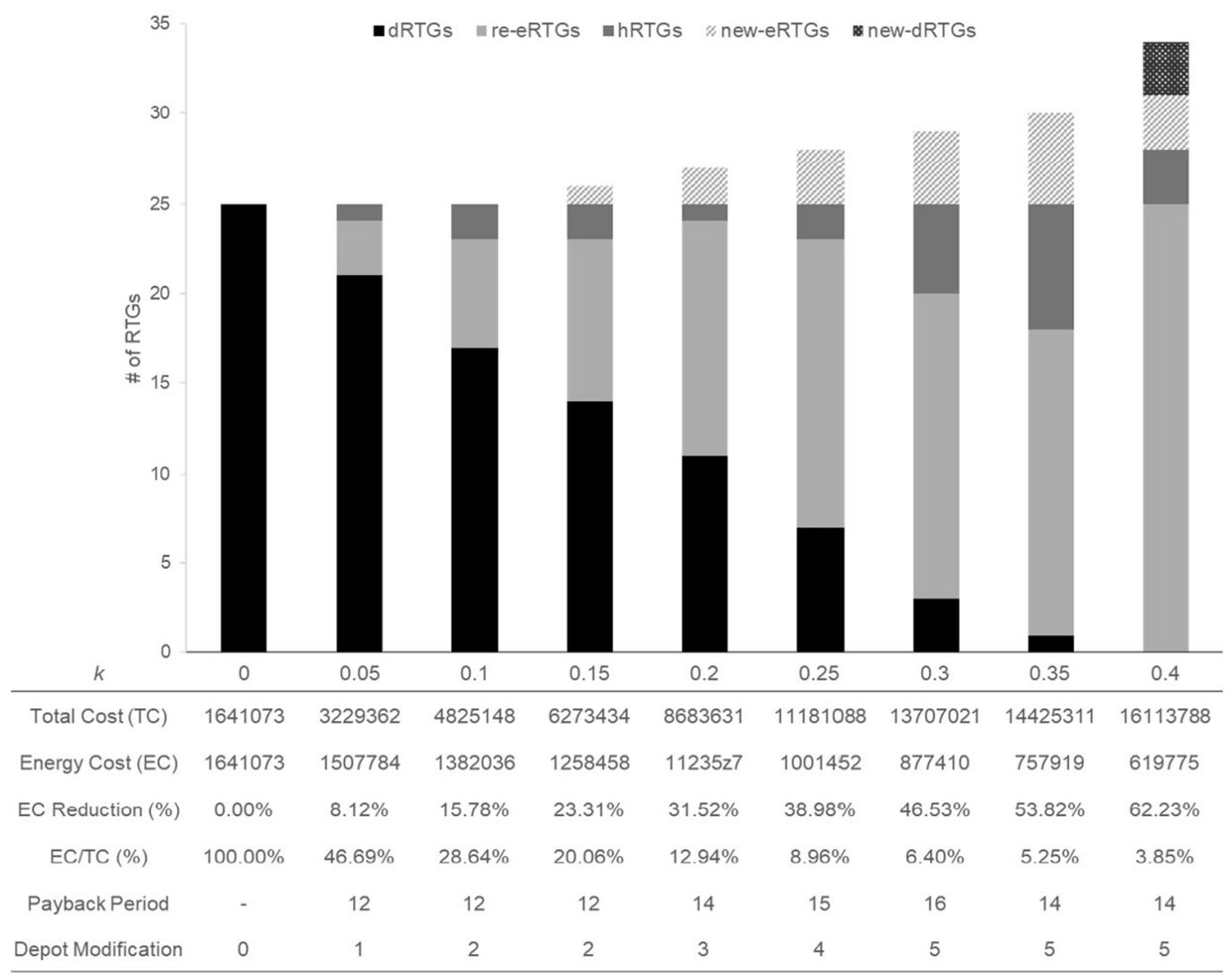

Fig. 4. Cost-effective strategies with various $\mathrm{CO}_{2}$ emission reduction targets.

Table 10

Cost-effective strategies with various eRTG operation capabilities.

\begin{tabular}{cccccc}
\hline$\beta_{2}$ & 3900 & 4800 & 6000 & 7000 & 8000 \\
\hline dRTGs $^{2}$ & 7 & 8 & 11 & 13 & 15 \\
re-eRTGs $^{1}$ & 16 & 16 & 13 & 11 & 10 \\
hRTGs $^{2}$ & 2 & 1 & 1 & 1 & 0 \\
new-eRTGs & 3 & 0 & 0 & 0 & 0 \\
new-dRTGs & 0 & 0 & 0 & 0 & 0 \\
Total cost & 11181088 & 8333891 & 7651663 & 7204312 & 6834905 \\
Energy cost & 1001452 & 998563 & 989985 & 991737 & 996587 \\
EC Reduction (\%) & $38.98 \%$ & $39.15 \%$ & $39.67 \%$ & $39.57 \%$ & $39.27 \%$ \\
EC/TC (\%) & $8.96 \%$ & $11.98 \%$ & $12.94 \%$ & $13.77 \%$ & $14.58 \%$ \\
Payback period & 15 & 10 & 9 & 9 & 8 \\
depot modification & 4 & 4 & 4 & 4 & 4 \\
eRTGs workload & $71.03 \%$ & $73.15 \%$ & $73.85 \%$ & $73.44 \%$ & $75.38 \%$ \\
(\%) & & & & &
\end{tabular}

\footnotetext{
1 eRTGs retrofitted from dRTGs.

2 eRTGs purchased.

3 dRTGs purchased.
}

management considering the distinct characteristics of different types of RTGs. Therefore, the contribution of this paper is to fill in this gap. An integer programming model is proposed to help decision-makers make monthly deployment plan, as well as a RTGs purchase and retrofit strategy, considering both the terminal operating efficiency and emissions. This paper is also one of the successful applications of the Corridor Method.

The application of our proposed model is illustrated with a modestly sized container terminal yard. Electrifying dRTGs to curb emissions is confirmed to be effective in achieving a $\mathrm{CO}_{2}$ reduction target and reducing energy costs. And an appropriate reduction target should be selected to make a trade-off with limited resources. A strict emissions limit requires significant expenditure and not always has merit financially. It is also found that the improvement of eRTG operating capability contributes to a shorter payback period, which illustrates the incentive to improve eRTG technologies to make themselves more attractive. The numerical results also confirm that Cplex solution time increases rapidly with problem size. The tailored Corridor Method (CM) effectively addresses this difficulty and obtains high-quality solutions 
Table 11

Performance of the CM algorithm ( $k=0.25, \mu=1.25)$.

\begin{tabular}{|c|c|c|c|c|c|c|c|c|c|}
\hline \multicolumn{4}{|c|}{ Problem size } & \multicolumn{2}{|c|}{ Cplex } & \multicolumn{2}{|c|}{$\mathrm{CM}$} & \multicolumn{2}{|c|}{ Time (s) } \\
\hline$N_{Z}$ & $N_{B}$ & $N_{I}$ & Rows-Columns-Nonezeros & Obj. & Gap (\%) & Obj. & RE (\%) & Cplex & $\mathrm{CM}$ \\
\hline 2 & 22 & 243 & 247-261-1053 & $4,693,110$ & 0.00 & $4,693,110$ & 0.00 & 2 & 34 \\
\hline 3 & 33 & 397 & $365-430-1774$ & $6,580,677$ & 0.00 & $6,580,677$ & 0.00 & 2 & 32 \\
\hline 4 & 44 & 573 & $475-617-2453$ & $8,567,513$ & 0.00 & $8,567,513$ & 0.00 & 4 & 39 \\
\hline 5 & 55 & 771 & $585-826-3176$ & $11,181,088$ & 0.00 & $11,181,088$ & 0.00 & 103 & 124 \\
\hline 6 & 66 & 991 & 695-1057-3943 & $12,452,377$ & 2.50 & $12,453,677$ & 0.01 & 322 & 356 \\
\hline 7 & 77 & 1233 & 805-1310-4754 & $15,399,224$ & 5.00 & $14,522,589$ & 0.06 & 813 & 561 \\
\hline 8 & 88 & 1497 & 915-1585-5609 & $16,412,059$ & 5.30 & $16,408,501$ & -0.02 & 5,433 & 617 \\
\hline 9 & 99 & 1783 & $1025-1882-6508$ & $18,526,527$ & 6.10 & $18,403,450$ & -0.66 & 83,235 & 818 \\
\hline 10 & 110 & 2091 & $1135-2201-7451$ & - & - & $20,621,875$ & - & - & 2,618 \\
\hline Average & - & - & - & - & - & - & - & $11,239.25$ & 578 \\
\hline
\end{tabular}

within a reasonable time.

In summary, the paper provides a theoretical foundation for crane operation supervisors and planners to make integrated cost-minimizing strategies of RTGs retrofit and deployment with emission reduction target and yard workload considered. However, there also exist some limitations in this study. To make the model more practical, the following issues can be further explored. First, random factors and uncertainties (fluctuation of workload, fuel prices, RTGs lifetime, and damage of RTGs) need to be further studied, because demand uncertainty is a prominent attribute of port terminal activities. Besides, this paper only focuses on minimizing the total cost. Future research might experiment with cost-benefit analysis.

\section{CRediT authorship contribution statement}

Yi Ding: Conceptualization, Resources, Methodology, Validation, Supervision. Yang Yang: Methodology, Data curation, Software, Writing - original draft. Leonard Heilig: Methodology, Validation, Writing - review \& editing. Eduardo Lalla-Ruiz: Methodology, Writing - review \& editing. Stefan Voss: Methodology Writing - review \& editing.

\section{Declaration of Competing Interest}

The authors declare that they have no known competing financial interests or personal relationships that could have appeared to influence the work reported in this paper.

\section{Acknowledgements}

We would like to express our gratitude to all the employees of Shanghai ShangDong International Container Terminal (SDICT) for their full support in this Research. We are especially grateful to Mr. Liu Chang Man, Senior Manager of the SDICT, for his valuable suggestions concerning modeling and Analysis. This project is partially supported by the National Natural Science Foundation of China [No. 71301101, No. 51409157], the National Key Research and Development Program of China [No. 2019YFB1704400, No. 2019YFB1704405].

\section{References}

Ansaripoor, A. H., Oliveira, F. S., \& Liret, A. (2014). A risk management system for sustainable fleet replacement. European Journal of Operational Research, 237(2), $701-712$.

Caserta, M., \& Voß, S. (2013). A mip-based framework and its application on a lot sizing problem with setup carryover. Journal of Heuristics, 19(2), 295-316.

Caserta, M., Ramirez, A., \& Voß, S. (2010). A math-heuristic for the multi-level capacitated lot sizing problem with carryover. In C. Di Chio, A. Brabazon, G. A. Di Caro, M. Ebner, M. Farooq, A. Fink, J. Grahl, G. Greenfield, P. Machado, M. O'Neill, E. Tarantino, \& N. Urquhart (Eds.), Applications of Evolutionary Computation (pp. 462-471). Berlin: Springer.

Caserta, M., Voß, S., \& Sniedovich, M. (2011). Applying the corridor method to a blocks relocation problem. OR Spectrum, 33(4), 915-929.

China's National Bureau of Statistics (2015) China Energy Statistics Yearbook 2014.
Chinese State Council (2016) The Work Plan for Controlling Greenhouse Gas Emission during the 13th Five-Year Plan Period. Tech. rep.

Environmental Protection Agency (2010) Clean Ports USA: Navigating Toward Cleaner Ports. Accessed 16 June 2017. https://nepis.epa.gov/Exe/ZyPDF.cgi/P100ABRE. PDF?Dockey $=$ P100ABRE.PDF.

ESPO (2012) ESPO Green Guide: Towards excellence in port environmental management and sustainability. Tech. rep., European Sea Ports Organization, URL: https://www. espo.be/media/espopublications/espo_green\%20guide_october\%202012_final.pdf.

Flynn, M. M., McMullen, P., \& Solis, O. (2007). High-speed flywheel and motor drive operation for energy recovery in a mobile gantry crane. In Proceedings of the 22th Annual IEEE Applied Power Electronics Conference and Exposition (APEC) (pp. 1151-1157).

Flynn, M. M., Mcmullen, P., \& Solis, O. (2008). Saving energy using flywheels. IEEE Industry Applications Magazine, 14(6), 69-76.

Geerlings, H., Kuipers, B., \& Zuidwijk, R. (2018). Ports and Networks: Strategies, Operations and Perspectives. London: Routledge.

He, J., Huang, Y., \& Yan, W. (2015). Yard crane scheduling in a container terminal for the trade-off between efficiency and energy consumption. Advanced Engineering Informatics, 29(1), 59-75.

Hsu, C. I., Li, H. C., Liu, S. M., \& Chao, C. C. (2011). Aircraft replacement scheduling: A dynamic programming approach. Transportation Research Part E: Logistics and Transportation Review, 47(1), 41-60.

Huang, S. Y., Guo, X., \& Lau, M. M. (2011). A three-level hierarchical workload management scheme for yard cranes in container terminals. In J. W. Böse, H. Hu, C. Jahn, X. Shi, R. Stahlbock, \& S. Voß (Eds.), Computational Logistics (pp. 221-232). Berlin: Springer.

Jinru, C. (2011). Energy saving after "electric instead of fuel" in Tianjin port. Science and Technology of Ports, 9, 48-49.

Kim, K. Y., \& Kim, K. H. (2003). Heuristic algorithms for routing yard-side equipment for minimizing loading times in container terminals. Naval Research Logistics (NRL), 50 (5), 498-514.

Kleindorfer, P. R., Neboian, A., Roset, A., \& Spinler, S. (2012). Fleet renewal with electric vehicles at La Poste. Interfaces, 42(5), 465-477.

Kuilboer, R. (2006). Revolutionary new drive design for rubber tired gantry's. Maxwell: Periodiek der Electrotechnische Vereeniging, 9(1), 12-14.

Liang, C. J., Gu, T. Y., Lu, B., \& Ding, Y. (2015). Genetic mechanism-based coupling algorithm for solving coordinated scheduling problems of yard systems in container terminals. Computers \& Industrial Engineering, 89, 34-42.

Linn, R. J., \& Zhang, C. (2003). A heuristic for dynamic yard crane deployment in a container terminal. IIE Transactions, 35(2), 161-174.

Lyu, Z. (2016). Concept design of hybrid crane vessel. Master's thesis. Delft University of Technology.

Ng, W., \& Mak, K. (2005). Yard crane scheduling in port container terminals. Applied Mathematical Modelling, 29(3), 263-276.

Obata, K., Sakamoto, T., Yoshioka, N., Fujisawa, N., Yoshikawa, H., \& Monzen, T. (2010). Grid-powered electric transfer crane. Mitsubishi Heavy Industries Technical Review, 47, $15-18$.

Parthanadee, P., Buddhakulsomsiri, J., \& Charnsethikul, P. (2012). A study of replacement rules for a parallel fleet replacement problem based on user preference utilization pattern and alternative fuel considerations. Computers \& Industrial Engineering, 63(1), 46-57.

PEMA Environment Committee (2013) Electrification of RTG Cranes in Ports and Terminals. Accessed 16 June 2017. https://www.pema.org/download/4866/.

Port Technology (2017) Vahle's Port of Felixstowe eRTG project wins big. Accessed 15 May 2018. https://www.porttechnology.org/news/vahles_port_of_felixstowe_ertg_ project_wins_big.

Port Technology (2018) SSA Terminals transform diesel RTGs. Accessed 15 October 2018. https://www.porttechnology.org/news/ssa_terminals_transform_diesel_rtgs.

Sha, M., Zhang, T., Lan, Y., Zhou, X., Qin, T., Yu, D., \& Chen, K. (2017). Scheduling optimization of yard cranes with minimal energy consumption at container terminals. Computers \& Industrial Engineering, 113, 704-713.

Shi, N., Song, H. Q., \& Powell, W. B. (2014). The dynamic fleet management problem with uncertain demand and customer chosen service level. International Journal of Production Economics, 148, 110-121.

Sniedovich, M., \& Voß, S. (2006). The corridor method: A dynamic programming inspired metaheuristic. Control \& Cybernetics, 35(3), 551-578. 
Stasko, T. H., \& Gao, H. O. (2010). Reducing transit fleet emissions through vehicle retrofits, replacements, and usage changes over multiple time periods. Transportation Research Part D: Transport \& Environment, 15(5), 254-262.

Stasko, T. H., \& Gao, H. O. (2012). Developing green fleet management strategies: Repair/retrofit/replacement decisions under environmental regulation. Transportation Research Part A: Policy and Practice, 46(8), 1216-1226.

Vujicic, A., Zrnic, N., \& Jerman, B. (2013). Ports sustainability: A life cycle assessment of zero emission cargo handling equipment. Journal of Mechanical Engineering, 59(9), $547-555$.

Yang, Y. C., \& Chang, W. M. (2013). Impacts of electric rubber-tired gantries on green port performance. Research in Transportation Business \& Management, 8, 67-76.
Yu, D., Li, D., Sha, M., \& Zhang, D. (2019). Carbon-efficient deployment of electric rubber-tyred gantry cranes in container terminals with workload uncertainty. European Journal of Operational Research, 275(2), 552-569.

Zhang, C., Yw, Wan, Liu, J., \& Linn, R. J. (2002). Dynamic crane deployment in container storage yards. Transportation Research Part B: Methodological, 36(6), 537-555.

Zhang, C., Zhang, Z. H., Zheng, L., \& Miao, L. (2011). A decision support system for the allocation of yard cranes and blocks in container terminals. Asia-Pacific Journal of Operational Research, 28(6), 803-829.

Zhao, N., Schofield, N., \& Niu, W. (2017). Energy storage system for a port crane hybrid power-train. IEEE Transactions on Transportation Electrification, 2(4), 480-492. 